\title{
Les recherches sur le bien-être animal : buts, méthodologie et finalité
}

\author{
I. VEISSIER ${ }^{1}$, C. BEAUMONT ${ }^{2}$, F. LEEVY3 \\ ${ }^{1}$ INRA, UR1213 Herbivores, F-63122 Saint-Genès Champanelle, France \\ 2 INRA, UR83 Recherches Avicoles, F-37380 Nouzilly, France \\ 3 INRA, CNRS, Université de Tours, Haras Nationaux, UMR85 Physiologie de la Reproduction et des Comportements, \\ F-37380 Nouzilly, France \\ Courriel : veissier@clermont.inra.fr
}

\begin{abstract}
Le réseau Agri Bien-être Animal, créé par l'INRA en 1998 à l'initiative de Robert Dantzer, permet à l'ensemble des chercheurs travaillant sur le thème du bien-être de mettre en commun leur expertise, leurs projets et leurs résultats. Ce regroupement apparaît particulièrement important en raison de l'approche nécessairement pluridisciplinaire du bien-être, rassemblant biologie, sciences sociales et sciences humaines. Le réseau est aujourd'hui constitué de 78 chercheurs INRA et 58 chercheurs d'autres organismes. Il mène principalement une action d'animation scientifique sous forme de séminaires (annoncés sur le site web http://www.tours.inra.fr/BienEtre) dont les comptes-rendus sont résumés dans

oiseaux (Arnould et Leterrier, Michel et al, Guéméné et al), des lapins (Mirabito), des ruminants (Mounier et $a l$ ) ou des porcs (Salaun et $a l$ ). Enfin une troisième partie traitera de questions appliquées communes aux différentes espèces, à savoir l'influence du stress prénatal sur le développement émotionnel de l'animal adulte et ses conséquences sur le bien-être (Roussel et $a l$ ), les rapports entre bien-être et santé animale (Bareille) et enfin les problèmes de bien-être animal relatif à la période qui précède l'abattage (Terlouw et al).

\section{1 / Pourquoi s'intéresser au bien-être animal ?} la lettre d'information (publiée sur le même site). Ce numéro spécial se fait l'écho des principaux travaux menés en France par les membres du réseau Agri Bien-être Animal. Il ne saurait être exhaustif mais présente les principaux résultats et pistes de réflexion. Un premier ensemble d'articles abordera 1'origine du concept de bien-être et ses principales définitions (Larrère), la caractérisation des émotions ressenties par l'animal (répertoire émotionnel) lesquelles déterminent sa capacité à ressentir du bien-être ou du mal-être (Boissy et $a l$ ), le sens que les éleveurs attribuent à ce concept en fonction de la relation qu'ils entretiennent avec leurs animaux (Dockès et Kling), ainsi que les relations entre les indicateurs comportementaux et physiologiques du mal-être (Richard et al). Dans une seconde partie, les atteintes au bien-être animal susceptibles d'être rencontrées dans les principales espèces d'élevage ainsi que les solutions pratiques envisagées ou mises en place seront abordés par type de production, qu'il s'agisse des poissons (Prunet et Aupérin), des
Même si le degré exact de sensibilité de chaque espèce animale est peu connu, c'est de la reconnaissance de la sensibilité des animaux - au moins pour certains d'entre eux dont les vertébrés que découlent les lois de protection de l'animal. Il en est ainsi de la loi dite welfare of farmed animals» (juin 2005). par la question du bien-être animal. «nature» du 10 juillet $1976^{1}$, laquelle stipule que «tout animal étant un être sensible doit être placé par son propriétaire dans des conditions compatibles avec les impératifs biologiques de son espèce». De ce fait, il est interdit d'exercer des «mauvais traitements envers les animaux domestiques ainsi qu'envers les animaux sauvages apprivoisés ou tenus en captivité» et nécessaire de «leur éviter des souffrances lors des manipulations inhérentes aux diverses techniques d'élevage, de parcage, de transport et d'abattage» (articles 214-1 et 214-3 du Code Rural). De même, au niveau de l'Union Européenne, en 1999, le traité d'Amsterdam² a fait passer les animaux d'un statut de biens marchands à celui d'êtres sensibles.

Cette prise en compte de la protection animale par les institutions répond aux attentes de nos sociétés. Si celles-ci sont exprimées plus particulièrement par les associations de protection animale, elles semblent partagées par une majorité de la population (figure 1).

Figure 1. Résultat de l'eurobaromètre 229 «Attitudes of consumers towards the

\section{Quand vous achetez de la viande, pensez-vous au bien-être des animaux?}

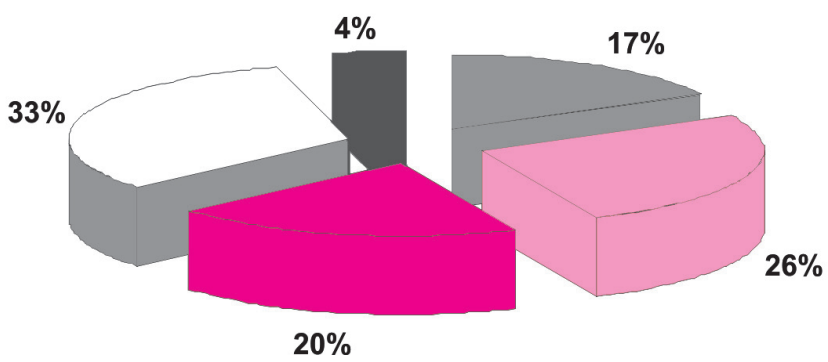

toujours

parfois

rarement

jamais

n'en achète pas

Près de la moitié des personnes ayant répondu au questionnaire semblent directement concernées

\footnotetext{
$1 \mathrm{http} / / /$ www.admi.net/jo/loi76-629.html et http://lexinter.net/Environnement/loi_relative_a_la_protection_de_la_nature.htm

2 http://europa.eu.int/eur-lex/fr/treaties/dat/amsterdam.html\#0001010001
} 
Ainsi, dans $65 \%$ des réponses à une consultation de la Commission Européenne réalisée en 2005 par Internet $^{3}$, le niveau de bien-être des animaux d'élevage est-il considéré comme «faible à très faible» dans l'Union Européenne. De même $75 \%$ des réponses expriment le souhait que des mesures soient prises pour mieux protéger les animaux, en particulier les poules pondeuses et les poulets de chair ( $76 \%$ des réponses) et les porcs (72\%), ce pourcentage étant plus faible pour les bovins $(63 \%)^{4}$. La France se situe généralement au niveau de la moyenne des 25 états de l'Union Européenne. La méthode retenue pour ce questionnaire peut être critiquée car les personnes qui se sentent concernées par la protection animale sont plus enclines à apporter leurs réponses. Toutefois les résultats confirment ceux précédemment obtenus par Ouedraogo (1998) et Latouche (1999), lesquels suggéraient qu'une majorité des français était favorable à la prise en compte du bien-être des animaux dans les élevages.

Les filières de production ont d'ores et déjà commencé à répondre à ces attentes. Leurs initiatives visent d'une part à revaloriser l'image des élevages et d'autre part à mobiliser les éleveurs sur le concept de bien-être animal. Il en est ainsi de la charte des bonnes pratiques en élevage initiée par les professionnels de l'élevage. Au Royaume-Uni et dans les pays d'Europe du nord, où les populations sont plus sensibles à la question du bien-être animal, ces initiatives ont démarré plus tôt. C'est par exemple, le cas des Freedom Food au Royaume-Uni, fondés en 1994 par la Royal Society for the Prevention of Cruelty against Animals. Elles sont également plus fréquentes. Ainsi, au Royaume-Uni, les éleveurs sont quasiment tous affiliés à un programme de certification (Veissier et al accepté).

La pertinence de ces initiatives dépend de la qualité de l'évaluation du bien-être et de l'analyse des facteurs influençant le bien-être d'un animal, lesquels dépendent de son espèce ou de son niveau de développement.

\section{2 / De la protection animale à l'étude du bien-être ani- mal}

La question sociétale de la protection des animaux vivant sous la dépendance de l'homme a trouvé son écho dans les milieux scientifiques où elle a été traduite en questions de recherche. La première étape a consisté à préciser la définition du bien-être, cet objet que les mesures de protection animale visent à atteindre. Parmi les nombreuses définitions proposées, Hughes (1976) le définit comme un état d'harmonie entre l'animal et son environnement, aboutissant à la complète santé mentale et physique mais il ne précise pas pleinement la notion d'harmonie. Broom (1987) propose de partir du concept de «coping» 5 (de l'anglais «je fais avec»), qu'il utilise dans le sens d'adaptation. Si l'adaptation à l'environnement peut être réalisée à moindre coût, par exemple sans dépenses énergétiques importantes, alors le niveau de bien-être sera élevé. Si au contraire, l'individu doit puiser de manière importante sur ses réserves pour s'adapter (par exemple, si la température extérieure est trop basse), alors le niveau de bien-être sera faible. Cette conception est souvent utilisée par les physiologistes. Dans la continuité des travaux de Selye (1936), ceux-ci considèrent que le stress (réponse de l'organisme à une agression quelle qu'elle soit, donc synonyme d'absence de bien-être) est une réponse d'adaptation détectée notamment par l'activation de la branche orthosympathique du système nerveux autonome et de l'axe corticotrope. Or ces réactions ne sont déclenchées que si l'animal perçoit comme une menace la situation dans laquelle il se trouve. Ainsi, des singes réagissent à un jeûne par une libération de cortisol dans la circulation générale alors que cette réponse fait défaut si la mise à jeun se fait à leur insu, par exemple en distribuant des aliments très peu nutritifs mais d'aspect similaire aux aliments normaux (Mason 1971). Cet auteur en avait conclu que l'on ne pouvait résumer le stress à une adaptation physiologique mais qu'il fallait prendre en compte la manière dont l'animal se représente son environnement Si ce dernier lui apparaît comme satisfaisant, l'animal sera en état de bien-être. S'il perçoit son environnement comme non idéal mais qu'il peut s'y ajuster, c'est un sentiment de «coping» qu'il éprouvera. Enfin, s'il perçoit son environnement comme désagréable et incontrôlable, ce sont des émotions négatives, de la souffrance, qui seront ressenties (figure 2).

Le bien-être est intimement lié à l'existence d'états mentaux dont les émotions ${ }^{6}$ constituent le point de départ. De fait, alors que les plantes ont des capacités d'adaptation, personne ne viendrait à parler de leur bien-être puisque, dépourvues de système nerveux, elles ne peuvent éprouver d'émotions. Par ailleurs, les attentes sociétales de protection animale partent bien $\mathrm{du}$ principe que les animaux sont des êtres sensibles qui peuvent donc souffrir (cf. supra), la sensibilité renvoyant à la fois aux capacités sensorielles (perception par les sens) et cognitives (traitement de l'information). Aussi le bienêtre est-il désormais considéré comme une expérience subjective de l'animal, un état mental, correspondant à l'absence d'émotions négatives (peur, frustration, douleur, faim ou soif prolongées...) et vraisemblablement à la présence d'émotions positives (confort, plaisir...) (Dawkins 1983, Duncan 2005). Cette définition dépasse la notion d'adaptation : si les émotions peuvent être considérées comme des processus d'adaptation qui interviennent dans la réaction de l'individu, à la fois en terme de comportement et de réponse physiologique (comme, par exemple, le stress qui permet de mobiliser des réserves énergétiques), ce sont les émotions qui constituent le cœur du bien-être et non les fonctions qu'elles sont censées protéger (Duncan 2002).

\section{3 / De la définition du bien- être aux recherches condui- tes pour améliorer les conditions de vie des ani- maux}

Une grande partie des recherches conduites dans le domaine du bien-être animal visent à développer des indicateurs de bien-être (ou au contraire de mal-être) et à évaluer le niveau de bienêtre permis par les conditions de vie des animaux. Les indicateurs de bien-être sont variés car le bien-être n'est pas un

\footnotetext{
3 http://europa.eu.int/comm/public opinion/archives/ebs/ebs 229 en.pdf

$4 \mathrm{http}: / /$ europa.eu.int/comm/food/consultations/action_plan_farmed_background_fr.htm

5 concept introduit par des psychologues

6 Une émotion est une réponse affective intense face à une situation, qui découle de l'interprétation de cette situation (Dantzer 1986)
} 
Figure 2. Perception de l'environnement par l'animal et bien-être.
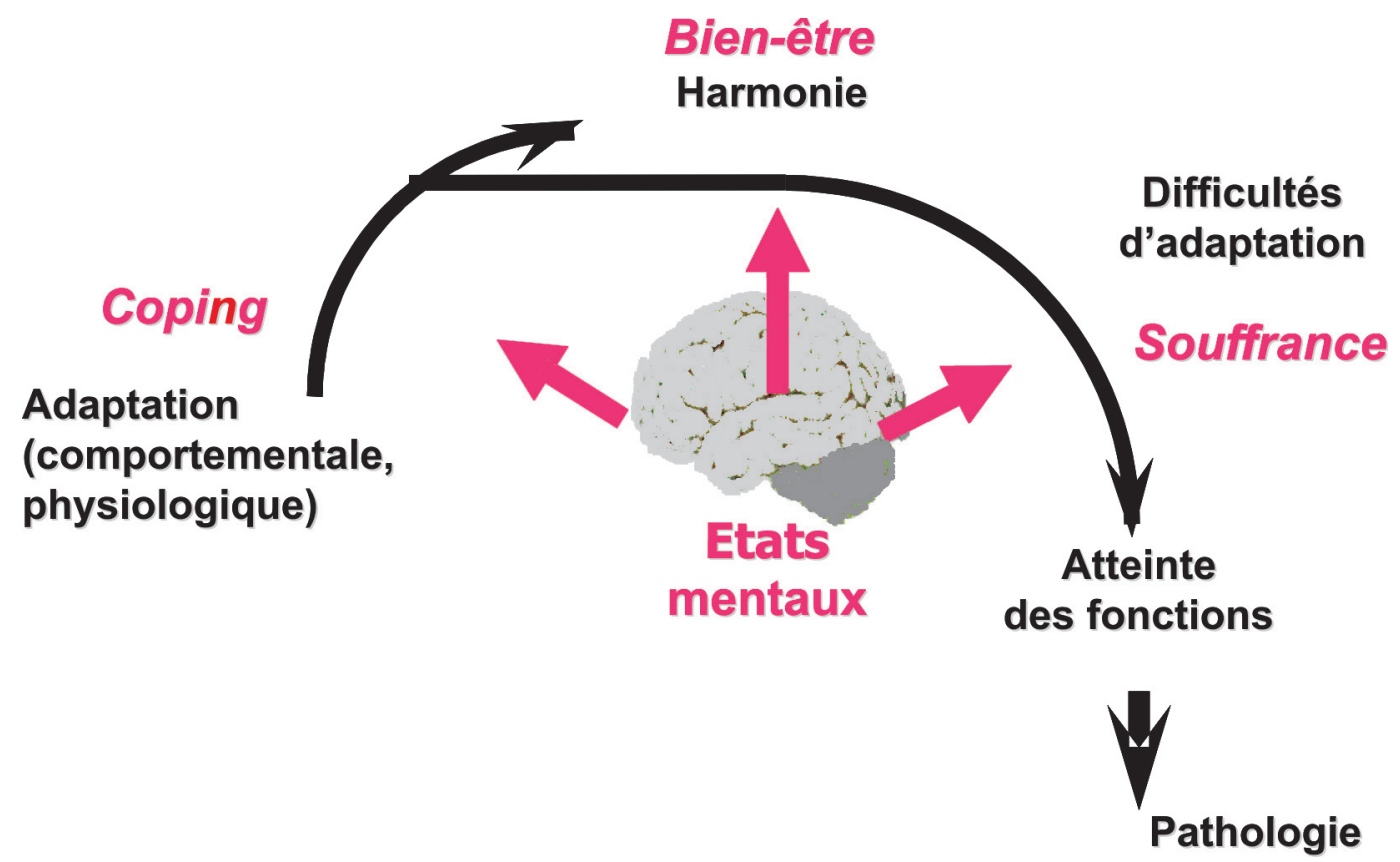

Le bien-être d'un animal est un état mental qui dépend de la façon dont cet animal perçoit son environnement. S'il perçoit que l'environnement satisfait toutes ses motivations, alors l'état mental sera le bien-être. Si l'environnement n'est pas idéal mais que l'animal perçoit qu'il pourra s'y adapter, alors l'état mental correspondra à un sentiment de «coping» («je fais avec»). Si au contraire l'animal perçoit qu'il a des difficultés pour s'adapter, alors la souffrance peut s'installer, les fonctions biologiques pourront être perturbées (modification de l'organisation des comportements et du fonctionnement des systèmes physiologiques impliqués dans le stress, diminution de l'état général pouvant aller jusqu'à l'apparition de pathologies).

concept unitaire. Il est au contraire formé de dimensions indépendantes.

Ainsi le Farm Animal Welfare Council (1992) liste cinq principes (ou «libertés» selon la définition anglaise) qui doivent être respectés pour atteindre le bien-être :

- l'absence de faim et de soif ;

- le confort physique ;

- la bonne santé et l'absence de blessure ou de douleurs ;

- la possibilité d'exprimer le comportement normal de l'espèce ;

- l'absence de peur et de détresse.

Ces cinq principes constituent la base de la majorité des réglementations visant à garantir le bien-être des animaux en élevages (voir par exemple le site de l'Union européenne sur la protection animale). Ils sont indépendants. Leur non-respect entraîne des symptômes spécifiques. Si la faim prolongée se traduit par la maigreur, l'impossibilité d'exprimer un comportement peut conduire à des activités anormales telles des stéréotypies, etc. Il est donc illusoire d'essayer de trouver l'indicateur universel du bien-être. En pratique, les travaux qui visent à apprécier le degré de mal-être d'un animal incluent :

- des indicateurs comportementaux en particulier l'expression d'activités anormales liée à l'impossibilité d'exprimer un comportement pour lequel l'animal est fortement motivé, une réactivité émotionnelle exacerbée ou diminuée sous l'effet d'un stress chronique...,

- des indicateurs physiologiques, comme la modification du fonctionnement de l'axe corticotrope sous l'effet d'un stress chronique,

- des indicateurs zootechniques, comme le gain de poids, la production de lait ou d'œufs qui peuvent varier, entre autres, si les animaux subissent un stress chronique,

- des indicateurs sanitaires, qui rendent compte de la présence de maladies et/ou de blessures lesquelles peuvent entraîner un malaise ou des douleurs pour l'animal.

Par ailleurs, les préférences des animaux appréciées au travers de tests de choix multiples permettent d'évaluer les préférences en posant directement la «question» à l'animal (Veissier et al 1999, figure 3).

L'appréciation des conditions d'élevage au regard du bien-être animal suit généralement la démarche suivante : dans un premier temps, les systèmes sont décrits puis les points critiques sont identifiés au regard des connais- sances préalables dont on dispose sur la biologie de l'espèce (besoins des animaux, éléments susceptibles d'être perçus négativement par l'animal). Ce premier temps correspond également aux approches européennes (cf. rapports scientifiques de l'European Food Safety Authority (EFSA)). Dans un deuxième temps, des expérimentations ou des enquêtes en ferme - suivant une approche épidémiologique - sont réalisées afin d'identifier les causes ou facteurs de risque. Enfin des améliorations sont proposées et testées. Par exemple, dans les années 1990, des travaux ont été conduits sur les systèmes de production des veaux de boucherie. A cette époque, la plupart des veaux de boucherie français étaient maintenus en cases individuelles de taille réduite et recevaient une alimentation exclusivement liquide. Plusieurs points critiques qui pouvaient menacer le bien-être ont ensuite été identifiés parmi lesquels la restriction des mouvements, l'absence de contacts sociaux, de tétée (la plupart des veaux étant nourris au seau) et du comportement alimentaire propre aux ruminants (ingestion de fourrages et rumination) qui apparaît dès les premières semaines de vie si l'animal a accès à des aliments solides). Leurs conséquences sur l'animal ont ensuite été évaluées. Ainsi l'absence d'aliment solide entraîne l'apparition d'activités orales non alimentaires telles que des 
Figure 3. Test de préférences de luminosité chez les dindons.

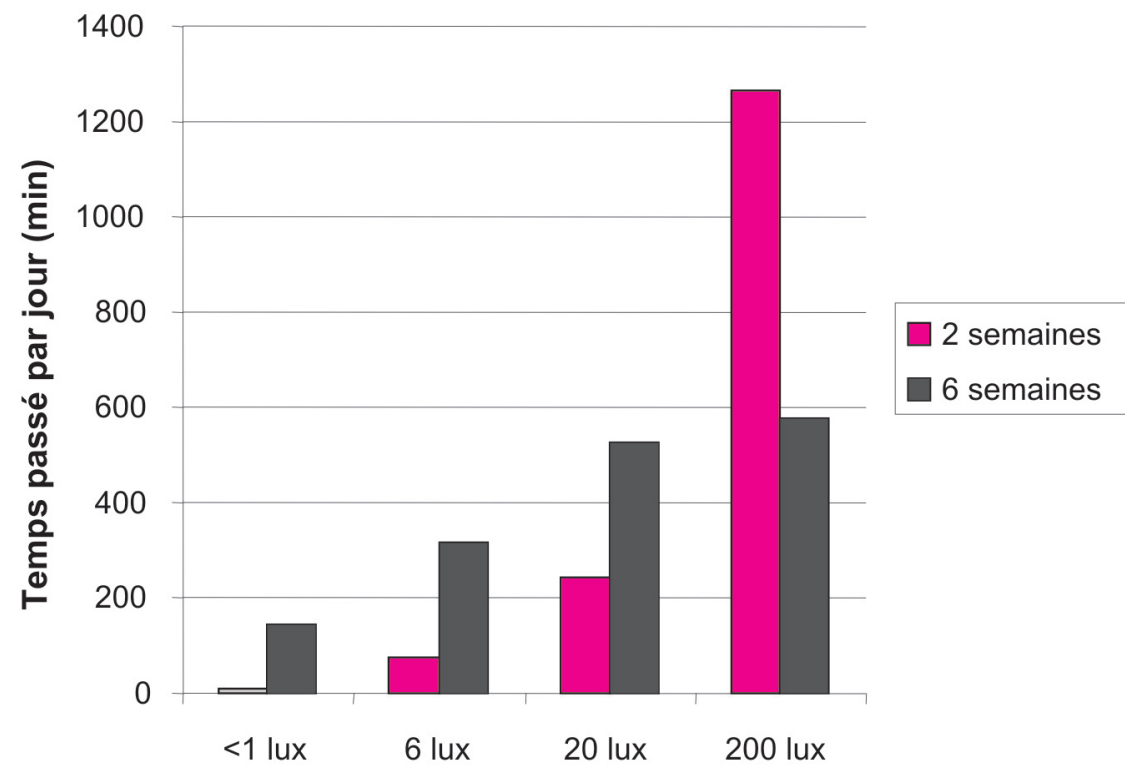

Les animaux ont en permanence le choix entre un compartiment quasiment obscur (< 1 lux), un compartiment dont la luminosité correspond à ce qui est couramment rencontré dans les élevages (6 lux), un compartiment dont la luminosité correspond à celle recommandée par la RSPCA (Royal Society for the Prevention of Cruelty to Animals, 20 lux) et un compartiment plus fortement éclairé (200 lux). Les dindons semblent éviter les compartiments peu éclairés. A deux semaines d'âge, ils présentent une nette préférence pour le compartiment fortement éclairé (Barber et al 2004).

grignotages des parois. Les solutions proposées ont consisté à ne plus pratiquer de séparation visuelle des veaux en cases individuelles, à élever les veaux en groupe et à distribuer des aliments solides en complément des aliments d'allaitement (tableau 1).

\section{4 / Le bien-être animal : contraintes ou atouts pour les élevages ?}

Lorsque l'on parle de bien-être animal, les élevages intensifs sont bien souvent critiqués car, ayant pour objectif premier la productivité de l'animal, ils ne tiennent pas forcement compte des contraintes qu'ils peuvent imposer à l'animal. Ainsi, l'instauration des quotas de production de lait dans les années 1980 a entraîné une augmentation du prix du veau «de 8 jours». De façon à limiter les répercussions de ce coût, la durée d'engraissement des veaux laitiers destinés à la boucherie a été allongée. Faute d'un agrandissement des cases individuelles dans lesquelles étaient maintenus les veaux, celles-ci se sont révélées trop étroites. Elles ne permettaient pas aux animaux de se coucher en allongeant leurs membres (Le Neindre 1993) et interdisaient tout mouvement, entraînant par là même une surexpression de l'activité locomotrice lorsqu'on libérait les veaux dans un espace plus large, surexpression qui signe une frustration antérieure (Dellmeier et al 1985). La situation des veaux a été améliorée grâce aux directives européennes 91/629 et 97/2/EC (Commission européenne 1991, Commission européenne 1997) : les veaux sont en cases collectives à partir de 8 semaines au minimum; en deçà de cet âge, ils peuvent être en cases individuelles mais celles-ci doivent être au moins aussi larges que la hauteur au garrot des animaux. De même, les animaux très productifs présentent une sensibilité accrue à certaines maladies: les vaches laitières hautes productrices sont plus sujettes aux mammites (Scientific

Tableau 1. Comparaison d'aliments utilisés pour la complémentation solide des veaux de boucherie réalisée sur la base de critères de comportement (activités orales non nutritives telles que les grignotages de parois), de santé (ulcères de la caillette), de fonctionnement biologique (poids du rumen et couleur des papilles (non présenté ici) utilisée comme révélateur de la fonction de rumination) et de production (gain de poids et couleur des carcasses).

\begin{tabular}{|c|c|c|c|c|c|}
\hline & $\underset{(\%)}{\text { Activités orales }^{a}}$ & $\begin{array}{l}\text { Poids du } \\
\text { rumen }^{b}\end{array}$ & $\begin{array}{l}\text { Ulcères de la } \\
\text { caillette }^{b}\end{array}$ & $\begin{array}{c}\text { Gain de } \\
\text { poids }{ }^{\mathrm{a}}(\%)\end{array}$ & $\begin{array}{l}\text { Couleur de } \\
\text { la carcasse }\end{array}$ \\
\hline Foin (ad libitum) & -80 & $x 1,7$ & pas d'effet & +12 & + foncée \\
\hline Paille hachée & -65 & $x 1,3$ & $\times 3$ & pas d'effet & pas d'effet \\
\hline Bouchons de paille & -55 & non mesuré & pas d'effet & pas d'effet & pas d'effet \\
\hline Ensilage maïs épi & pas d'effet & $x 1,5$ & $\times 3$ & +9 & + foncée \\
\hline Ensilage de maïs & -70 & $\times 1,5$ & $\times 2$ & +6 & pas d'effet \\
\hline Ensilage de maïs séché & pas d'effet & non mesuré & $\times 2$ & +8 & pas d'effet \\
\hline Pulpe betterave & pas d'effet & non mesuré & $\times 2$ & +6 & + foncée \\
\hline Avoine aplatie & pas d'effet & non mesuré & pas d'effet & pas d'effet & pas d'effet \\
\hline Pellets $11,6 \%$ Cellulose brute & pas d'effet & $x 1,3$ & pas d'effet & +9 & + foncée \\
\hline Pellets $21,2 \%$ Cellulose brute & non mesuré & $x 1,2$ & pas d'effet & +10 & pas d'effet \\
\hline Pellets $26 \%$ Cellulose brute & non mesuré & $x 1,4$ & pas d'effet & +5 & pas d'effet \\
\hline $\begin{array}{l}\text { Pellets } 1,6 \% \text { Cellulose brute } \\
\text { puis paille hachée }\end{array}$ & -40 & non mesuré & pas d'effet & +13 & pas d'effet \\
\hline
\end{tabular}

a : \% par rapport à des témoins recevant uniquement un aliment d'allaitement.

b : facteur de multiplication par rapport aux témoins recevant uniquement de l'aliment d'allaitement.

Ces travaux ont permis de proposer aux producteurs d'utiliser une supplémentation alimentaire sous forme de concentrés et d'aliments riches en cellulose qui offre un bon compromis entre les objectifs de réduction des comportements oraux, limitation des ulcères et maintient de la qualité des carcasses (dernière ligne du tableau) (Veissier et al 2003). 
Committee on Animal Health and Animal Welfare 1999) et les troubles locomoteurs du poulet de chair semblent liés à leur vitesse de croissance (Arnould et al 2007). Des études sont en cours pour préciser l'importance de la génétique, à la fois dans ces caractères de résistance aux maladies et dans les critères d'adaptation comportementale des animaux à leurs conditions d'élevage, le tout dans le but de concilier bien-être et aptitudes économiques de l'animal (Beaumont et al 2002).

Mais, bien qu'ils soient souvent considérés comme socialement plus acceptables, les élevages extensifs peuvent aussi présenter des inconvénients pour les animaux. La mortalité peut être plus élevée, en particulier chez les agneaux nouveau-nés élevés sur partiques se détériorent. Si les conditions dans lesquelles sont élevés les animaux permettent, dans la très grande majorité des cas, un bon état général des animaux et par suite une bonne production, les optima de production et de bien-être ne se situent pas toujours au même point.

En retour, le niveau de bien-être peut avoir des conséquences sur la production. Tel est le cas des mammites qui entraînent une chute de la production laitière d'autant plus élevée que les vaches sont fortes productrices (Lescourret et Coulon 1994). De même, les animaux d'élevage, qui appartiennent à des espèces grégaires, tissent des liens entre congénères familiers (entre mère et cours lorsque les conditions clima-

jeune, puis entre animaux de même âge) ; la séparation de partenaires familiers et le mélange avec d'autres animaux entraînent à la fois des réponses de stress et des chutes de performance, de croissance chez les jeunes et de production laitière chez les mères (Hasegawa et al 1997, Price et al 2003, Veissier et al 1989b). En retour, une amélioration des conditions de vie peut augmenter la productivité par animal. Ainsi pour leur couchage, les vaches préfèrent un matelas à une logette seulement recouverte de paille hachée (Bony 2000) ; elles produisent alors jusqu'à un litre de lait supplémentaire par jour. Ainsi, dans bien des cas, l'amélioration du bien-être s'accompagne d'une augmentation de la productivité par animal. Cependant, elle peut aussi entraîner une augmentation des charges de l'éleveur, comme c'est le cas des coûts de production d'œufs plein-air, de $40 \%$ plus élevés que ceux d'œufs produits en cage «batterie» (Magdelaine et Mirabito 2003).

Bien-être animal et production sont donc deux objectifs distincts mais non opposés, obéissant chacun à leur logique. A l'heure actuelle où les activités agricoles sont remises en cause par bon nombre de citoyens (cf. $\$ 1$ ) et où les éleveurs ont des difficultés à maintenir leur revenu, il est indispensable de trouver des solutions alliant bien-être animal et productivité. Dans bien des cas, cela est possible. Ainsi, l'apport d'une complémentation solide au régime essentiellement liquide (lait ou aliment d'allaitement) des veaux de boucherie a été rendu obligatoire par la réglementation afin que les

Figure 4. Schéma général du système visé par le projet Welfare Quality ${ }^{\circledR}$.

\section{Consommateurs Distributeurs}

\section{Producteurs}

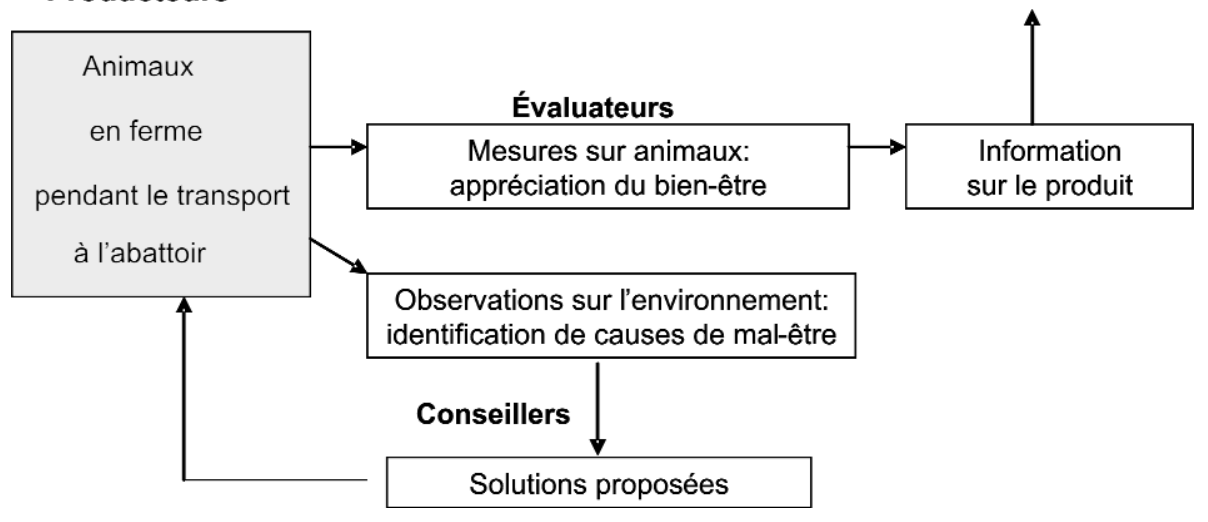

Des mesures de comportement, de santé... sont réalisées sur les animaux dans les élevages, au cours du transport ou de l'abattage afin d'évaluer le niveau général de bien-être des animaux. Cette évaluation donne lieu à une information sur les produits (lait, viande, œuf), qui est transmise au distributeur et au consommateur. Dans le cas où le niveau de bien-être est jugé insuffisant, des observations sont réalisées sur l'environnement afin d'identifier les causes de mal-être et des solutions pourront être proposées aux producteurs. La méthode d'évaluation du bien-être et l'information retranscrite aux consommateurs seront standardisées au niveau européen (Veissier et al 2005). veaux expriment leur comportement alimentaire (Directive 91/629 amendée par 97/2/EC). Cet apport diminue nettement la fréquence des activités orales non alimentaires (comme le grignotage des parois) mais il s'accompagne souvent d'ulcères de la caillette. Désormais, en combinant aliments fibreux et aliments riches en glucides rapidement fermentescibles, il est possible de réduire les activités orales non alimentaires des veaux et d'éviter les ulcères tout en augmentant le poids des carcasses de 10 à $20 \mathrm{~kg}$ (Veissier et al 2003). De même la réduction du stress à l'abattage est bénéfique non seulement au bien-être de l'animal mais également à la maturation des viandes (Terlouw et al 2007). Enfin des critères de sélection touchant au bien-être ont été inclus dans des schémas de sélection : c'est le cas par exemple de la propension aux mammites (Sandøe et Christensen 1998) ou aux troubles locomoteurs des poulets de chair (Beaumont et Chapuis 2004).

Ajoutons à cela que le respect du bienêtre animal peut être valorisant pour les éleveurs. Ainsi l'abandon, à la suite de la directive 97/2/EC, des cases individuelles des veaux de boucherie semble avoir entraîné une revalorisation de leur métier pour les éleveurs de cette filière qui disent retrouver un vrai métier d'éleveur (Dockès et Kling 2007).

Au niveau européen, un label spécifique pourrait permettre d'harmoniser les pratiques visant à garantir au consommateur que les produits qu'ils achètent ont été produits dans le respect du bien-être tout en garantissant au producteur un prix d'achat plus élevé (Commission Européenne 2002). Les critères d'évaluation du bien-être des animaux dans un élevage ou un abattoir ainsi que les moyens nécessaires à la mise en place d'une telle certification sont explorés dans le projet européen Welfare Quality ${ }^{\circledR}$ (Blokhuis et al 2003) (http://www.welfarequality.net/ everyone, figure 4$)$.

\section{En conclusion : A quoi servent les recherches sur le bien-être animal ?}

Les recherches sur le bien-être des animaux d'élevage servent avant tout à apporter des arguments objectifs au débat de société sur la protection de ces animaux. Les résultats scientifiques contribuent à l'élaboration des textes visant à protéger les animaux. Ainsi, au sein du Conseil de l'Europe, les repré- 
sentants de sociétés savantes (Fédération Européenne de Zootechnie FEZ ou EAAP (European Association for Animal Production), Société Internationale d'Ethologie Appliquée (ISAE)) participent aux discussions du comité permanent pour la convention de protection des animaux dans les élevages. De même, au niveau de l'Union Européenne, lorsque la commission (en particulier la Direction Générale de santé et de protection des consommateurs ou DG-SANCO) décide de prendre des mesures dans un domaine particulier de la protection animale (tel que le transport des animaux, l'élevage des veaux,...), elle consulte le comité scientifique vétérinaire, devenu par la suite comité scientifique pour la santé animale et le bien-être animal et qui fait désormais partie de l'Autorité Européenne de Sécurité des Aliments (AESA ou EFSA pour European Food Safety Authority). A chaque question de la DG-SANCO, le comité forme un groupe de travail ad hoc formé de scientifiques reconnus dans le domaine à analyser (par exemple des scienti- fiques ayant travaillé sur le veau si la question porte sur le bien-être des veaux). Ce groupe fait la revue de l'existant (méthodes d'élevage, d'abattage etc.) et évalue l'impact des facteurs d'élevage sur le bien-être de l'animal. Les conditions de logement, d'alimentation, de soins, etc. font l'objet d'une attention particulière. Le groupe de travail émet un avis sur les risques qu'il pense devoir être traités en priorité et peut recommander des mesures précises. Ces avis s'appuient sur les données scientifiques disponibles, en particulier celles publiées dans des revues à comité de lecture. Mais ces dernières sont parfois en nombre réduit, en particulier pour les espèces ou productions secondaires. Il est donc important de la part des scientifiques d'anticiper les questions du législateur en augmentant les données scientifiques (voir articles par espèces).

Les résultats permettent également de conseiller les éleveurs soucieux de bien produire en respectant le bien-être des animaux (voir plus haut les exemples de solutions proposées). Toutefois, dans l'état actuel des choses, ce conseil sur le bien-être animal auprès des éleveurs est encore peu développé. A l'avenir cet aspect devra être renforcé de sorte que les travaux de recherche aillent jusqu'au transfert des résultats. Il s'agit là d'un enjeu essentiel puisque seul le choix de mesures appropriées permettra non seulement d'améliorer le bien-être des animaux mais aussi de garantir la viabilité des élevages tout en améliorant leur acceptabilité par la société.

\section{Remerciements}

$\mathrm{Au}$ nom de l'ensemble du réseau $A G R I B E A$, les auteurs tiennent à remercier Robert Dantzer pour son apport considérable à la problématique du bien-être animal, tant au niveau des initiatives que des idées. Ils remercient également la direction de l'INRA, et plus particulièrement la direction scientifique Animal et Produits Animaux, pour leur soutien.

\section{Références}

Arnould C, Leterrier C., 2007, Bien-être animal en élevage de poulets de chair. INRA Prod. Anim., 20(1), 41-46.

Barber C.L., Prescott N.B., Wathes C.M., Le Sueur C., Perry G., 2004. Preferences of growing ducklings and turkey poults for illuminance. Anim. Welf., 13, 211-224.

Beaumont C., Chapuis H., 2004. Génétique et sélection avicoles : évolution des méthodes et des caractères. INRA Prod. anim., 17, 35-43.

Beaumont, C., Roussot, O., Marissal-Avry, N., Mormede, P., Prunet, P., Roubertoux, P., 2002. Génétique et adaptation des animaux d'élevage : introduction. INRA Prod. Anim., 15, 343-348.

Blokhuis H.J.J.R.B., Geers R., Miele M., Veissier I., 2003. Measuring and monitoring animal welfare: transparency in the food product quality chain. Anim. Welf., 12, 445-455.

Bony J.B.M., 2000. Comparaisons de différentes natures de couchage pour les vaches laitières en logettes. Renc. Rech. Rum., 7, 82.

Broom D.M., 1987. Applications of neurobiological studies to farm animal welfare. In: Biology of stress in farm animals: an integrative approach, Wiepkema, P.R., Van Adrichem, P.W.M. (Eds.), Martinus Nijhoff Publishers, Dordrecht, Boston, Lancaster, UK, 101-110.

Commission Européenne, 1991. Directive 91/629/CEE du Conseil, du 19 novembre 1991, établissant les normes minimales relatives a la protection des veaux. Journal officiel, L 340, 28-32.

Commission Européenne, 1997. Directive 97/2/CE du Conseil du 20 janvier 1997 modifiant la directive 91/629/CEE établissant les normes minimales relatives à la protection des veaux. Journal officiel, L 25, 24-25.
Commission Européenne, 2002. Communication from the European commission to the council and the European parliament on animal welfare legislation on farmed animals in third countries and the implications for the EU, COM, 626.

Dantzer R., 1986. Les émotions. Presses Universitaires de France, 121p.

Dawkins M.S., 1983. La souffrance animale. Editions du Point Vétérinaire, Maisons-Alfort, $152 p$.

Dellmeier G.R., Friend T.H., Gbur E.E., 1985. Comparison of four methods of calf confinement. II. Behavior. J. Anim. Sci., 60, 1102-1109.

Dockès A.C., Kling-Eveillad F., 2007. les représentations de l'animal et du bien-être animal par les éleveurs français. INRA Prod. Anim. 20(1), 23-28.

Duncan I.J.H., 2002. Poultry welfare: Science or subjectivity? Br. Poult. Sci., 43, 643-652.

Duncan I.J.H., 2005. Science-based assessment of animal welfare: farm animals. Rev. Sci. Tech. Off. Int. Epiz., 24, 483-492.

Farm Animal Welfare Council, 1992. FAWC updates the five freedoms. Vet. Rec., 17, 357.

Hasegawa N., Nishiwaki A., Suguwara K.,Ito I., 1997. The effects of social exchange between two groups of lactating primiparous heifers on milk production, dominance order, behavior and adrenocortical response. Appl. Anim. Behav. Sci., 51, 15-27.

Hughes, B.O., 1976. Behaviour as an index of welfare. Proc. $5^{\text {th }}$ Eur. Poultry Conf., 1005-1018.

Latouche K., 1999. French people's willingness to pay for farm animal welfare. Kunisch M., Eckel H (Eds), Int. Cong., 108-112.
Le Neindre P., 1993. Evaluating housing systems for veal calves. J. Anim. Sci., 71, 13451354.

Lescourret F., Coulon J.B., 1994. Modeling the impact of mastitis on milk production by dairy cows. J. Dairy Sci., 77, 2289-2301.

Magdelaine P., Mirabito L., 2003. Le bien-être animal: atout ou contrainte? Sci. Techn. Avic., Hors Série, 51-56.

Mason J.W., 1971. A re-evaluation of the concept of non-specificity in stress theory. J. Psychiatric Res., 8, 323-333.

Mignon-Grasteau S., Faure J.M., 2002. Génétique et adaptation : le point des connaissances chez les volailles. INRA Prod. Anim. 15(5), 367-364.

Ouedraogo A., 1998. Ethical consumers? Social representations of stock farming in France. Veissier I., Boissy A. (Eds). Proc. 32 ${ }^{\text {nd }}$ Cong. Int. Soc. Applied Ethol., 204.

Price E.O., Harris J.E., Borgwardt R.E., Sween M.L., Connor J.M., 2003. Fenceline contact of beef calves with their dams at weaning reduces the negative effects of separation on behavior and growth rate. J. Anim. Sci., 81, 116-121.

Sandøe P., Christensen L.G., 1998. Staying good while playing god: the ethics of breeding farm animals. Veissier, I., Boissy, A. (Eds). Proc. $32^{\text {nd }}$ Cong. Int. Soc. Applied Ethol., 180.

Scientific Committee on Animal Health and Animal Welfare, 1999. Report on animal welfare aspects of the use of bovine somatotrophin. European Commission, 91p.

Sely, H., 1936. A syndrome produced by diverse nocuous agents. Nature, 32 . 
Terlouw E.M.C., Arnould C., Auperin B., Bern C., Le Bihan-Duval E., Lefevre F., Lensink J., Mounier L., 2007. Impact des conditions de pré-abattage sur le stress et le bien-être des animaux d'élevage. INRA Prod. Anim., 20(1), 92-100.

Veissier I., Le Neindre P.,Trillat G., 1989 b. Adaptability of calves during weaning. Biol. Comport., 14, 66-87.
Veissier I., Sarignac C., Capdeville J., 1999. Les méthodes d'appréciation du bien-être des animaux d'élevage. INRA Prod. Anim., 12, 113-121.

Veissier I., Bertrand G., Toullec R., 2003. Le veau de Boucherie : Concilier bien-être animal et production. INRA Editions, coll. Du labo au terrain, Versailles, France, 231p.

Veissier I., Blokhuis H.J., Geers R., Jones R.B., Miele M., 2005. Le projet Welfare Quality: de l'attente des consommateurs à la mise en place de certifications bien-être en élevage. Bull. Acad. Vét. Fr., 158, 263-267.

Veissier I., Butterworth A., Bock B., Roe E., 2007. European approaches to ensure animal welfare. Appl. Anim.Behav. Sci., 100, Specia issue: What's new in Europe since the Brambell report, accepté.

\section{Résumé}

La protection des animaux est inscrite dans la réglementation française et européenne. Elle répond à une attente sociétale majeure dans nos pays occidentaux, attente qui part du principe que les animaux que nous utilisons sont des êtres vivants sensibles à qui l'homme doit assurer un minimum de bien-être. La façon dont un animal appréhende son environnement va influencer son état de bien-être et ce, positivement si l'environnement satisfait ses besoins et ses motivations, mais négativement si l'environnement est perçu comme menaçant, désagréable. Le niveau de bien-être d'un animal est apprécié au travers de nombreuses observations portant sur son comportement, l'état d'activation des systèmes physiologiques, et son état général. Les conditions d'élevage, généralement choisies en fonction d'objectifs de production, peuvent également influencer le bien-être d'un animal. En retour, un bien-être dégradé peut entraîner une moindre productivité. Aussi des solutions d'élevage permettant de concilier production et bien-être animal doivent-elles être mises au point. Ce numéro spécial vise à mieux cerner la notion de bien-être animal et rassemble les principaux travaux entrepris par les membres du réseau $A g r i$ Bien-être Animal dans les différentes productions. Les résultats des recherches en bien-être animal sont utilisés lors de l'élaboration de réglementations. Ils doivent également servir au conseil en élevage.

\section{Abstract}

\section{Research into animal welfare: aims, methods and purpose}

Animal protection is now included in French and European legislation. It meets a major concern of western societies that stems from the acknowledgement that animals used by humans are sentient beings that should be guaranteed a minimum protection. The way animals perceive their environment determines their well-being: it will be good if the animal perceives that the environment meets its needs, while it will be low for an animal that perceives its environment as threatening or unpleasant. To assess the welfare state of an animal, many behavioural, physiological and biological indices need to be taken into account. The living conditions of farm animals are often chosen according to production objectives and may not always ensure a high level of animal welfare. At the same time, a low welfare state can have adverse effects on animal productivity. Hence remedial solutions that meet both production and welfare requirements should be developed. This special issue aims to refine the concept of animal well-being and will present a review of results of the research undertaken by the members of the AGRIBEA network. The findings can be used for drawing up regulations and advising farmers.

VEISSIER I., BEAUMONT C., LÉVY F., 2007. Les recherches sur le bien-être animal : buts, méthodologie et finalité. INRA Prod. Anim., 20, 3-10. 
